\title{
LEFT ATRIAL SHAPE AND FUNCTION AFTER ENDOVASCULAR DEVICE AND SURGICAL CLOSURE OF ASDS IN CHILDREN
}

\author{
Smorgon Andrey Vladimirovich ${ }^{1}$, Sokolov Alexandr Anatolievich ${ }^{1}$, Varvarenko Viktor \\ Ivanovich $^{1}$, and Krivoshchekov Evgeny Vladimirovich ${ }^{1}$ \\ ${ }^{1}$ FGBNU Tomskij nacional'nyj issledovatel'skij medicinskij centr Rossijskoj akademii nauk
}

February 27, 2022

\begin{abstract}
Retrospective analysis of echocardiograms was performed in 756 children who received endovascular device or surgical ASD closure from 2006 to 2016 in the Cardiac Center in Tomsk Russia. 564 patients had an endovascular closure and 192 had surgical correction. Follow-up duration was from 1 day to 10 years, mean 3.6 yrs for the device group and 4.2 yrs for the surgery group. The control group consisted of 3393 age-matched healthy patients. In patients with endovascular closure of an ASD, $35 \%$ had a change in the shape of the left atrium in early follow-up. Changes in the shape of the left atrium at early follow-up were more often observed in the device group and in children of a younger age. The left atrial changes were a decrease in sphericity and an increase in ellipsoidy. Changes in the shape of the left atrium persisted in $22 \%$ after transcatheter correction in the long-term. The change in shape of the left atrium after the placement of ASD devices was accompanied by activation of the mechanical function of the atrium and an increase in the filling pressure of the left ventricle. These changes were not accompanied by any disturbance in the contractility and volume of the heart chambers. In the group with surgical correction of ASD, the contractility and volume of the heart chambers did not significantly differ from those in the device closure group.
\end{abstract}

\section{LEFT ATRIAL SHAPE AND FUNCTION AFTER ENDOVASCULAR DEVICE AND SUR- GICAL CLOSURE OF ASDS IN CHILDREN}

Sokolov Alexandr Anatolievich MD ${ }^{1}$

Varvarenko Viktor Ivanovich $\mathrm{MD}^{1}$

Krivoshchekov Evgeny Vladimirovich $\mathrm{MD}^{1}$

Smorgon Andrey Vladimirovich $\mathrm{MD}^{1}$

Cardiology Research Institute, Tomsk National Research Medical Center, Russian Academy of Sciences

ADDRESS FOR CORRESPONDENCE:

634012, Russian, Tomsk, Kievskay st. 111-A, Cardiology Research Institute, Tomsk National Research Medical Center, Russian Academy of Sciences

Tel: +7 (3822) 55-83-67, +7 (3822) 55-36-10

CORRESPONDING AUTHOR:

Smorgon Andrey Vladimirovich MD, e-mail:sav.ssmu@gmail.com

634017, Russian, Tomsk, Andrey Kraychkov st.17-227 
Tel: +79069481206

\section{AUTHOR CONTRIBUTIONS}

All authors helped craft and critically revise themanuscript. All authors approved the final version of the manuscriptand can be held accountable for the integrity of the work.

\section{CONFLICT OF INTERESTS}

All authors declare that there are no conflict of interests.

FUNDING STATEMENT:

There is no funding for this study.

\section{DATA AVAILABILITY STATEMENT}

The data that support the findings of this study are available on rea-sonable request from the corresponding author. The data are not pub-licly available due to institutional data protection and ethical restrictions

Abstract

Retrospective analysis of echocardiograms was performed in 756 children who received endovascular device or surgical ASD closure from 2006 to 2016 in the Cardiac Center in Tomsk Russia. 564 patients had an endovascular closure and 192 had surgical correction. Follow-up duration was from 1 day to 10 years, mean 3.6 yrs for the device group and 4.2 yrs for the surgery group. The control group consisted of 3393 agematched healthy patients.

In patients with endovascular closure of an ASD, 35\% had a change in the shape of the left atrium in early follow-up. Changes in the shape of the left atrium at early follow-up were more often observed in the device group and in children of a younger age. The left atrial changes were a decrease in sphericity and an increase in ellipsoidy. Changes in the shape of the left atrium persisted in $22 \%$ after transcatheter correction in the long-term. The change in shape of the left atrium after the placement of ASD devices was accompanied by activation of the mechanical function of the atrium and an increase in the filling pressure of the left ventricle. These changes were not accompanied by any disturbance in the contractility and volume of the heart chambers. In the group with surgical correction of ASD, the contractility and volume of the heart chambers did not significantly differ from those in the device closure group.

Introduction

Atrial septal defects (ASDs) account for 10-15\% of all patients with congenital heart defects (CHD). Endovascular device closure is currently accepted as the treatment of choice in most patients with secundum ASD. Its efficacy and complication rates are comparable to surgery. However, in some patients who have contraindications for using an endovascular approach, surgical treatment is still needed. [1].

ASD occluder devices and surgically placed patches may increase the rigidity of the septum. These interventions, while eliminating the interatrial shunt, may limit septal mobility, and possibly disrupt the mechanical function of the left atrium (LA). The purpose of this study was to study the effect of surgical and endovascular device closure of ASDs on the size, shape and function of the LA and left ventricle (LV) in children.

Subjects

We retrospectively studied consecutive echocardiograms from children who received endovascular device or surgical ASD closure between 2006 and 2016 at the Cardiac Center in Tomsk, Russia. In total, 756 patients with ASD were evaluated. Seventy-five percent (564 patients) had endovascular device closure. Various devices were utilized (Nit-Occlud, Amplatzer, Occlutech), the LA side of the devices ranged from 14 to 38 $\mathrm{mm}(25.6+4.1)$. Twenty-five percent (192 patients) had surgical ASD closure with a pericardial patch. Follow-up duration was1 day to 10 years (yrs) (mean 3.6 yrs for the device group and 4.2 yrs for the surgery 
group). The control group consisted of 3393 healthy age-matched children. Patient demographics and followup intervals are demonstrated in Table 1 . The surgical group included patients with contraindications for endovascular correction, in most cases because of a deficient rim.

Methods

Institutional review board approval was obtained for analysis of the echocardiography database, with no requirement to obtain informed consent. International Review Board approval was not sought. The trial was not registered with Clinicaltrials.gov.

Echocardiographic images were obtained using commercially available ultrasound equipment (iE33, Philips Ultrasound). Standard echo measures of chamber sizes and volumes were evaluated and indexed to body surface area. These measurements were compared to previously published normative data. Doppler echocardiographic evaluation of mitral inflow velocities and A wave duration were used in the assessment of left ventricular diastolic function. E/A ratio transmitral flow and mean septal and lateral mitral annular tissue Doppler diastolic velocities and peak duration were evaluated. To reduce the effect of heart rate, diastole duration as a percentage of the duration of the R-R interval was used. Diastolic time expressed as percent of cardiac cycle (D\%) calculated by the formula: $\mathrm{D} \%=((\mathrm{R}-\mathrm{R})-\mathrm{ET}) / \mathrm{RR} \times 100$, where $\mathrm{D} \%$ - diastole duration, ET- ejection time. Diastolic timing intervals were measured from transmitral flow and named DF (diastole flow). Mitral annular tissue Doppler was named MD (mechanical diastole).

Left atrial shape index (LASI) was calculated as the ratio of the anteroposterior and transverse size to vertical: LASI $=(\mathrm{W} 1+\mathrm{W} 2) / 2^{*} \mathrm{H} * 100$. Where $\mathrm{W} 1=$ anteroposterior size measured in parasternal short axis, $\mathrm{W} 2$ = width measured in apical 4-chamber projection, and $\mathrm{H}=$ height of the LA measured from the posterior LA wall to the mitral annulus in the apical 4-chamber projection. (Fig.1). an increase in the index close to 100 was regarded as an increase in sphericity, a decrease in ellipsoidity. Left atrial force (LAEF) was determined by the Manning method $[5,6,7]$.

LAVi-left atrial volume index and RAVi-right atrial volume index were calculated as volume/BSA. Atrial volumes was calculated by standard methods using 4 and 2 chamber projections. An atrial index (Ai) was defined as RAV $\backslash \mathrm{LAV}$, using unindexed values. [8]

Since various size occluders were used, the ratio of the smallest size of the left atrium (w1) and the diameter of the LA side disc was calculated. LASI in the control group was evaluated by a frequency table. Only $5 \%$ of controls had an LASI $<65$ and we defined that as the "cut of point" value between normal and abnormal (Table 2, Fig. 2).

Continuous variables were presented as mean $+/$ - standard deviation. Categorical data were represented by frequencies and percentages. Clinical and procedural data were compared with paired and unpaired t-tests. Categorical data used applied tests that were two-sided. $\mathrm{P}<0.05$ was considered statistically significant.

Results

Patients in the surgical group had significantly larger left to right shunt $(\mathrm{Qp} / \mathrm{Qs})$ than patients in the device group $(2,97+0.47$ versus $2.10+0.64, \mathrm{p}=0.01)$. The RAVi in the surgical group was larger (Table 3$)$. During follow-up, the device group LASI was significantly decreased, and

LASI before device or surgical closure were similar but both were significantly different from the control group. Patients with ASD had LASI $<65$ in 10,6\% of cases (Fig.3). After closing the ASD, the percentage of patients who had an abnormal LASI $(<65)$ increased in both groups but was more pronounced in the device group (Fig.3) . During follow-up LASI was decreased from the first day to several years after device implantation. But, there was no significant difference between the surgery group and control group at late follow-up. The device group had a decreased LASI compared to controls (Fig.2). There was no significant change in the surgical group (Fig.2).

The number of patients with an abnormal shape of the LA in the surgical group decreased during the observation period. In the device group, LASI was stable from 6 months post-procedure throughout the 
follow-up period. (Fig.3). There was no difference between surgery and device groups in respect to chamber volumes, contractility, and right ventricular systolic pressure at all data collection points.

We then evaluated the possible significance of changing LASI to chamber volumes and function. All device patients were divided into two groups. A reshaped group defined as: (RS) - LASI [?] 65 any point after implantation, and a saved shape group that had an LASI > 65 (Table 5).

Chamber Volumes

At 1-5 days after ASD closure, LV volume increased significantly and right-sided chamber sizes decreased in both surgical and device groups. After ASD closure the following data were obtained: LVEDV and LVEF were unchanged in both groups, LA volume decreased significantly in the device group (Table 2) and RVSP and RA volumes decreased. The LAEF in the device group with "reshaped" LA remained maximal during short-term (1-5 days, mean 3,4+1,3) and long-term (2 -10 mean 3,6+3,5years) (Fig 4) follow-up.

Other echo parameters in these patients were not different between the surgery group and the patients who had "saved shape" group. Only mechanical diastole in the "reshape" subgroup was longer compared to controls (Table 4)

In the early period (1-5 days) after ASD closure in patients in both the surgical and device groups, the volume of the RA decreased significantly but the Ai was unchanged (Table 3). At the same time, the LVEDVi in the device group increased, while in the surgical group it decreased in comparison with the preoperative period. The RVSP in both groups decreased significantly $(\mathrm{p}<0.05)$ while the contractility of the LV remained unchanged. The patients in the device group with a increased LASI at the time of implantation of the device were significantly younger than patients with a preserved LASI $(2,06+0,47$ and $2,98+0,64$ yrs, $\mathrm{p}=0.02)$.

\section{Long-term follow-up}

The surgical and device closure groups were compared in long-term follow-up from 2.1 to 10 years. Patient age in the groups was the same $9.6+6.9$ years (surgery) and $9.0+4.5$ years (device). Mean follow-up in the surgery group was 4.3 years and 3.6 years in device the group.

The LASI was lower in the device group $(74,0+0,3)$ versus the surgery group $(70,0+2)$. A LASI $<65$ (significantly different from the norm) in the device group was found in $22,5 \%$, of the device group, $8.0 \%$ of the surgical group and $16.6 \%$ of the overall study cohort. The indexed LV diastolic volume in the groups did not differ (surgical: $49,9+/-10,8 \mathrm{ml} / \mathrm{m}^{\wedge} 2$; device: $49,3+/-9,6 \mathrm{ml} / \mathrm{m}^{\wedge} 2, \mathrm{p}=0.61$ ). Similarly, ejection fraction was the same in the surgical and device groups $(71 \%+/-5 \%)$ (Table 5$)$.

The LACF (left atrial ejection force) was significantly lower in the surgery group than in the device group (Figure 5). But, the contraction force of the LA in the device group did not differ from the control group.

It should be noted that in patients who lost form of the LA after ASD closure (34.4\% of total), the strength of the contraction of the LA was higher than the group average and amounted to $9.51+4.7 \mathrm{kdynes} / \mathrm{cm} / \mathrm{m}^{\wedge} 2$, while in those with impaired LA form after surgery, the strength of the contraction of the LA was not significantly different from the entire surgery group $\left(6.5+4.4\right.$ kdynes $\left./ \mathrm{cm} / \mathrm{m}^{\wedge} 2\right)$ or the control group $(6.6+$ $\left.4.8 \mathrm{kdynes} / \mathrm{cm} / \mathrm{m}^{\wedge} 2\right)$.

\section{Discussion}

In this study, $35 \%$ of children with device closure of an ASD had a change in the shape of the LA - a decrease in sphericity and an increase in ellipsoidity. The first mechanisms of LA remodeling after ASD closure, specifically changes in shape and force of contraction, may turn out to be unfavorable factors for the patient in the future. Similar changes were found in adult patients with interatrial adiposity. These remodeling processes were associated with increased LA global kinetic energy and ejection force at the hemodynamic level, which may serve to compensate for functional LA deterioration in asymptomatic patients [ 10]. It is hoped that these changes are not significant for the lives of our young patients. In the long-term after 
surgical and endovascular treatment of ASDs, the main hemodynamic parameters and indexed chamber volumes were not abnormal (Table 5).

It is interesting that loss of LA shape after ASD device closure was found in the youngest children. There are several problems encountered during device closure in small children. The inherent design of several commercially available devices, with excessive disc width, may increase the risk for erosion and cause the device to encroach on adjacent cardiac structures [11]. But the stiffness of these devices may have a detrimental effect on the LA shape. This topic has not been well researched. It is possible that device size or stiffness may restriction growth of the LA in the antero-posterior direction. It is controversial if interatrial septum length is a limiting factor for sizing the device. [12.]. Our data suggest that device oversizing may have a negative long-term effect on LA shape.

Patients in the device group with an increased LASI were significantly younger than patients with a preserved LASI $(2,06+0,47$ versus $2,98+0,64 \mathrm{yrs}$, respectively, $\mathrm{p}=0.02)$. The calculated ratio of the minimum size of the interatrial septum and the LA disc of the device had a significant negative correlation at 6 and 12 months of follow-up $(\mathrm{r}=-0.62$ and $-0.55, \mathrm{p}=0.01)$. This meant that the larger the device with respect to the size of the septum, the greater the change in the shape of the LA.

There is an opinion that ASD device occlusion should performed in infants and young children only if the disk diameter is smaller than the diameter of the atrial septum. [13.] In our study, we used large oversized devices and in small children this may change LA shape.

The increased left ventricular filling pressure after ASD closure should not be regarded as a negative factor. Most likely this was due to increased volume entering the left ventricle after ASD closure. It is not known if the initial mechanisms of LA remodeling, changes in shape and force of contraction, will turn out to be unfavorable factors in the future. Similar changes were found in adult patients with interatrial adiposity. These remodeling processes were associated with increased LA global kinetic energy and ejection force at the hemodynamic level, which may serve to compensate for those functional deteriorations of LA in asymptomatic patients [10].

At long-term follow-up, left ventricular filling pressure was normal in the device group. Recent studies have shown that LA dysfunction after ASD device closure is more common in children. In adult patients LA contractility improves after ASD closure [14]. In addition, the size of the ASD device was negatively correlated with contractility of the LA. These results imply that ASD occlusion devices adversely affect LA function and may be important in choosing other treatment strategies. Depression of LA function was detected by speckle tracking in adult patients immediately after device implantation, but this resolved by one month after intervention $[15,16]$. It is unknown if the changes in LA shape that we observed after ASD device closure impact the lives of young patients.

\section{Conclusions}

Endovascular device closure of ASDs was accompanied by a change in LA shape - a decrease in sphericity and an increase in ellipsoidity. These changes were more frequent in younger children. Changes in LA shape persisted in $22 \%$ of patients after ASD device closure. The LA shape after ASD closure device placement was accompanied by activation of the mechanical function of the atrium and an increase in the filling pressure of the left ventricle. These changes were not accompanied by any disturbance in the contractility and volume of the heart chambers.

Reference

1. Jung SY, Choi JY. Transcatheter closure of atrial septal defect: principles and available devices. J Thorac Dis. 2018 Sep;10(Suppl 24): S2909-S2922. doi: 10.21037/jtd.2018.02.19

2. Bisbal F, Guiu E, Cabanas P et al Reversal of spherical remodelling of the left atrium after pulmonary vein isolation: incidence and predictors $\backslash \backslash$ Europace. 2014 Jun;16(6):840-7. doi: 10.1093/europace/eut385. Epub 2014 Jan 2 
3. Chung CS, Karamanoglu M, Kovács SJ. Duration of diastole and its phases as a function of heart rate during supine bicycle exercise// Am J Physiol Heart Circ Physiol. 2004 Nov;287(5):H2003-8. Epub 2004 Jun 24

4. Mondal T, Slorach C, Manlhiot C, et al. Prognostic implications of the systolic to diastolic duration ratio in children with idiopathic or familial dilated cardiomyopathy// Circ Cardiovasc Imaging. 2014 Sep;7(5):773-80. doi: 10.1161/CIRCIMAGING.114.002120. Epub 2014 Aug 19

5. Triposkiadis F, Harbas C, Sitafidis G, et al Echocardiographic assessment of left atrial ejection force and kinetic energy in chronic heart failure. $\backslash \backslash$ Int J Cardiovasc Imaging. 2008 Jan;24(1):15-22. Epub 2007 Mar 30. DOI:10.1007/s10554-007-9219-7

6. Manning WJ, Silverman DI, Katz SE, Douglas PS. Atrial ejection force: a noninvasive assessment of atrial systolic function. J Am Coll Cardiol. 1993 Jul;22(1):221-5.

7. Chinali M, de Simone G, Liu JE et al. Left atrial systolic force and cardiac markers of preclinical disease in hypertensive patients: The Hypertension Genetic Epidemiology Network (HyperGEN) Study \\Am J Hypertens. 2005 Jul;18(7):899-905

8. Pritchett AM, Jacobsen SJ, Mahoney DW, et al. Left atrial volume as an index of left atrial size: a population-based study// J Am Coll Cardiol. 2003 Mar 19;41(6):1036-43. DOI: 10.1016/s07351097(02)02981-9

9. Bisbal F, Guiu E, Calvo N, et. al. Left atrial sphericity: A new method to assess atrial remodeling. Impact on the outcome of atrial fibrillation ablation. $\backslash \backslash \mathrm{J}$ Cardiovasc Electrophysiol. $2013 \mathrm{Jul} ; 24(7): 752-9$. doi: 10.1111/jce.12116. Epub 2013 Mar 14.,

10. Lai YH, Yun $\mathrm{CH}, \mathrm{Su} \mathrm{CH}$ et al. Excessive interatrial adiposity is associated with left atrial remodeling, augmented contractile performance in asymptomatic population \\Echo Res Pract. 2016 Mar;3(1):5-15. doi: 10.1530/ERP-15-0031. Epub 2016 Jan 5

11. Kim NK, Park SJ, Choi JY.Transcatheter closure of atrial septal defect: does age matter? Korean Circ J. 2011 Nov;41(11):633-8. doi: 10.4070/kcj.2011.41.11.633. Epub 2011 Nov 29

12. Sharma B, Pinto R, Dalvi B. Transcatheter closure of atrial septal defect in symptomatic children weighing [?]10 kg: Addressing unanswered issues from a decade of experience// Ann Pediatr Cardiol. 2020 Jan-Mar;13(1):4-10. doi: 10.4103/apc.APC_66_19. Epub 2019 Dec 4.

13. Gao W, Zhou AQ, Yu ZQ et al. Transcatheter closure of secundum atrial septal defect in children//Zhonghua Er Ke Za Zhi. 2004 Apr;42(4):287-90

14. Salvo G., Drago M, Pacile Get al. Atrial Function After Surgical and Percutaneous Closure of Atrial Septal Defect: A Strain Rate Imaging Study// J Am Soc Echocardiogr , 18 (9), 930-3 Sep 2005 PMID: 16153516 DOI: $10.1016 /$ j.echo.2005.01.029

15. Cakal S, Eroglu E, Baydar O et al Two-dimensional strain and strain rate imaging of the left atrium and left ventricle in adult patients with atrial septal defects before and after the later stage of percutaneous device closure// Echocardiography.2015 Mar;32(3):470-4. doi: 10.1111/echo.12693. Epub 2014 Jul 24

16. Suzuki K, Kato T, Koyama S Et al. Influence of Percutaneous Occlusion of Atrial Septal Defect on Left Atrial Function Evaluated Using 2D Speckle Tracking Echocardiography. doi: 10.1536/ihj.19-173. Epub 2020 Jan 17.

\section{Hosted file}

figure (1).docx available at https://authorea.com/users/422679/articles/557978-left-atrialshape-and-function-after-endovascular-device-and-surgical-closure-of-asds-in-children

\section{Hosted file}


table (1).docx available at https://authorea.com/users/422679/articles/557978-left-atrialshape-and-function-after-endovascular-device-and-surgical-closure-of-asds-in-children 